\title{
Withanolides: Strategies for Enhanced Production and Mechanistic Insights into their Mode of Action
}

\author{
ABHINAV GROVER* \\ School of Biotechnology, Jawaharlal Nehru University, New Delhi 110 067, India
}

(Received on 19 May 2014; Revised on 20 January 2015; Accepted on 06 April 2015)

\begin{abstract}
A large number of withanolides are isolated from solanaceous plants. Around 360 naturally occurring withanolides have been identified till date. These compounds are found to have useful pharmacological activities, out of which, anticancer activities of withanolides have drawn considerable interest from researchers. As plants being their sole source, in which they are synthesized in considerably low amounts, biotechnological interventions have been explored in order to increase the yield of these secondary metabolites. Their molecular mechanisms of action also remained largely unexplored for a long time. This review covers recent attempts which have ben made to enhance the production of withanolides and discusses the studies that elucidated their prospective action mechanisms.
\end{abstract}

Key Words: Withanolides; Withania somnifera; Secondary Metabolites; Transformation; Overexpression; Squalene Synthase; Mechanism of Action

\section{Introduction}

Withanolides are naturally occuring plant secondary metabolites. Phytochemical studies have been mainly conducted on 19 genera of Solanaceae. These genera are predominantly found in temperate and tropical zones around the world. A number of withanolides have been characterized over the past few years. A total of 360 naturally occurring withanolides have been identified till date (Chen et al., 2011).

Withanolides possess different pharmacological properties such as antimicrobial, anti-inflammatory, anti-stress, anti-oxidant, immunoregulatory, antifeedant, insecticidal, trypanocidal and leishmanicidal (Mishra et al., 2000; Grover et al., 2013). Additionally, it also manifests antitumor, cardioprotective and neuroprotective activities. Besides withanolides, there are a number of phytocompounds listed in Table 1, which exhibit similar properties. Biological activity of withanolides is due to $\mathrm{C}_{28}$ side chain in which $\mathrm{C}-22$ and $\mathrm{C}-26$ are oxidized and are rearranged in a ergostane framework to form a six-membered lactone ring (Mirjalili et al., 2009). Structure-activity corelation of withanolides has been studied which has revealed that unsaturation at $\alpha, \beta$ position of ketone ring in ring $A$ and epoxy group at position $5 \beta, 6 \beta$ in ring $\mathrm{B}$ are necessary for withanolides to remain biologically active (Chen et al., 2011). During their work in 2004, Kinghorn and co-workers have identified a compound which possesses $\mathrm{C}_{27}$ skeleton, and shows cancer chemopreventive activity. They named it as norwithanolide which was found to be different from other withanolides because they contain a typical $\mathrm{C}_{28}$ backbone (Kinghorn et al., 2004).

Studies carried out by Chaurasiya and coworkers in 2012 have revealed that precursor molecules for biosynthesis of withanolides are isoprenoids. Classical cytosolic mevalonate (MVA) pathway and 2-C-methyl-D-erythritol-4-phosphate 
Table 1: List of various pharmacologically active compounds from plant sources

\begin{tabular}{ll}
\hline Anticancer metabolite & Plant source \\
\hline Argimonolide & Argimonia pilosa \\
Bruceantin & Brucea antidysenterica \\
Caesalpins & Bruguiera paviflora \\
Camptothecin & Camptotheca acuminita \\
Etoposide & Podophyllum pelatum \\
Flavopiridol & Dysoxylum binectariferum \\
Geranin & Geranium robertianum \\
Hydrocyanic acid & Cassia absus \\
Norwithanolides & Depreasubtriflora \\
Paclitaxel & Taxus brevifolia \\
Polyphenols & Camellia sinensis \\
Quercetin & Cajanus cajan \\
Selenium, ayanin & Physalis angulate \\
Taxol & Taxodium distichum \\
Vinblastine, vincristine & Catharanthus roseus \\
Withanolides & Withania somnifera \\
\hline &
\end{tabular}

(MEP) pathway localized in plastids, form 24methylene cholesterol which finally leads to the formation of isoprenoids (Chaurasiya et al., 2012). This key molecule, after various biochemical modifications including hydroxylation and glycosylation leads to the formation of different withanolides. A variety of withanolides are shown to be present in different plant parts. However, very few of them show tissue specific biosynthesis (Chaurasiya et al., 2009).

Biosynthesis of this group of secondary metabolites occurs in roots from where they get transported for accumulation in leaves (Sangwan et al., 2008). The concentration of these active phytocompounds is generally low in plants. It ranges from 0.001 to $0.5 \%$ of dry weight in roots and leaves of the plant. On the other hand, it is not economically feasible to chemically or synthetically produce withanolides because the process is highly expensive. Therefore, there is an indispensable need for development of commercially viable alternatives for producing high amounts of withanolides (Grover et al., 2013). As traditional cultivation of these plants is time consuming and laborious, various strategies have been employed to enhance the production of withanolides and these have been discussed in the following text.

\section{Strategies for Enhanced Production}

\section{Molecular Approach}

The biosynthetic pathway of withanolides is not fully characterized but isoprenoids are known to serve as precursors which later split into triterpene and sterol biosynthesis. The role of enzyme squalene synthase (SS) is well characterized for biosynthesis of sterol (Tozawa et al., 1999). Metabolic engineering based approaches were utilized to overexpress SS. Since SS has captivated considerable interest as a probable diverging point from isoprenoid pathway to sterol biosynthesis (Lee et al., 2002), it has been utilized as a central regulatory enzyme to enhance the withanolide content. Transformed cell lines of Withania somnifera were established in which the SS was overexpressed (Grover et al., 2013). They further measured the SS activity in transformed cells by quantifying the amount of NADPH consumed in transformation of Farnesyl Pyrophosphate (FPP) to squalene using fluorescence spectroscopy. This group obtained 4-fold increase in the activity of SS and 2.5fold increase in withanolide content (Grover et al., 2013).

\section{Hairy Root Cultures for the Production of Withanolides}

Withanolides are synthesized in tissue specific manner. Their concentration varies from one organ to another but largely these phytocompounds are present in roots and leaves. Kaiser et al. (2006) reported that this group of secondary metabolites is biosynthesized in roots from where it gets transported to leaves for accumulation. Studies on the biosynthesis of secondary metabolites in plants can be performed easily by Agrobacterium rhizogenes-mediated hairy root production (Murthy et al., 2008). Hairy roots are characterized by their extensive branching and rapid 
growth in the medium. They have the capability of being genetically stable, and have been used for the production of secondary compounds in cultures (Zhao et al., 2004; Santos et al., 2005). Various explants from seedling roots, stems, hypocotyls, cotyledons, cotyledonary nodes and leaf segments have been used to initiate the cultures. However, only cotyledons and leaf segments responded best by developing transformed roots. A 2.7 fold increase in withanolide content was observed in the transformed roots (Murthy et al., 2008).

\section{Elicitor Treatment for Increased Production of Withanolides}

Traditionally, cell and organ cultures have been used for production of secondary metabolites, but the yield of withanolides have been low. Attempts have been made to increase concentration of metabolites in shorter period of time. Exogenous addition of elicitors of biotic and abiotic origin is considered to be the most fruitful strategy for enhanced production of secondary metabolites (Radman et al., 2003). Another study proposed a hypothesis that these elicitor molecules act as extracellular or intracellular signals which, when perceived by the receptor present on plasma membrane, initiate a signalling cascade that is required for the activation or de novo biosynthesis of transcription factors (Zhao et al., 2005). These in turn regulate the expression of genes involved in plant secondary metabolism (Kim et al., 2009). Sivanandhan et al. (2013) used methyl jasmonate and salicylic acid (SA) as elicitors for Withania somnifera hairy root cultures (Sivanandhan et al., 2013). Withaferin A content was 34-fold higher than control with the elicitation of methyl jasmonate and its production was 42-fold higher with SA elicitation. Withanolide A production was 50-fold higher with MeJ treatment whereas it was 58-fold higher with SA treatment. However, 38-fold higher production of withanone was obtained with MeJ whereas 46-fold increase was obtained with SA.

\section{Pharmacological Mode of Action of Withanolides}

Herbal medicines contain withanolides as a major component and have a wide range of applications. These phytocompounds get accumulated in the roots and leaves of plants. Pharmacological investigations of these compounds have provided scientific support for traditional uses of many withanolide- producing plants. We provide a concise summary of the biological and pharmacological activities of naturally occurring withanolides and their possible mode of action.

\section{Activation of p53}

There are several mechanisms that elucidate the mode of actions of withanolides for their anticancer activities. p53 activation by withanolides makes them a potent tumour suppressor. p53 regulates cell cycle by holding it at $\mathrm{G}_{1} / \mathrm{S}$ phase and finally leads to apoptosis of cancer cells by forming nuclear Bax/p53 complexes and their interaction with other nuclear chaperons (Raffo et al., 2000). Most cancer cells exhibit high levels of Hsp70 proteins which help in tumour progression. Mortalin, a multifunctional protein, is an Hsp70 protein family member which interacts with $\mathrm{p} 53$. It has been reported that mortalin-p53 interaction is synchronized under stress conditions and occurs mainly in cancer cells in which p53 is heavily phosphorylated (Lu et al., 2011) as observed in Fig. 1. Withanone causes selective killing of cancer cells by energizing tumour suppressor p53 pathway. It impedes the mortalin-p53 complex origination resulting in the nuclear translocation and activation of p53 function. It is already reported that mutant mortalin lacking either carboxyl-terminal region or aminoterminal region could bind to MKT-077 but amino acid residues 252-310 are primarily required for attaching mortalin to MKT-077 (a cationic inhibitor of mortalin). Withanone can bind to mortalin in the region where p53 and MKT-077 binding sites are present and hence could release p53 from mortalin-p53 complex. After release p53 gets activated and starts functioning which subsequently leads to growth arrest or apoptosis (Grover et al., 2012).

\section{Activation - Suppression of NF- KB}

$\mathrm{NF}-\kappa \mathrm{B}$ is present principally in cytoplasm in the form of an inactive complex with I $\kappa \mathrm{B}$ inhibitor proteins. Signalling pathway leads to the activation of IKK (IKB kinase) a multisubunit complex, containing two kinase subunits, IKK $\alpha$ and IKK $\beta$, and a regulatory subunit NEMO (NF- $\kappa \mathrm{B}$ essential modulator) which then 


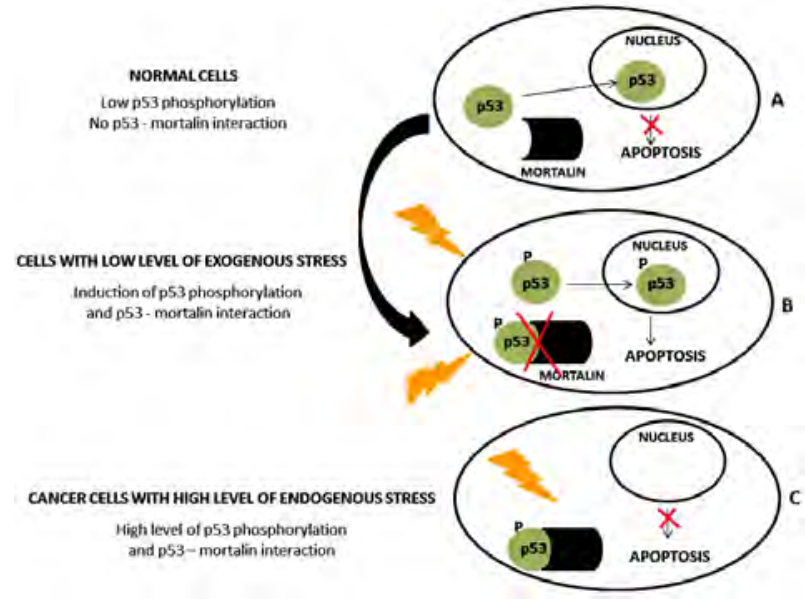

Fig. 1: A schematic model showing translocation of p53 in nucleus of a cell under different stress levels. (A) In normal and non-malignant cells with low p53 phosphorylation level, mortalin-p53 interaction does not occur, and hence free p53 gets shifted from cytoplasm to nucleus. However, unphosphorylated p53 is incapable of inducing apoptosis. (B) Exposure of cells to exogenous stress induces mortalin-p53 collaboration by $\mathbf{p 3}$ phosphorylation and amassing. If mortalin-p53 interaction is blocked by silencing mortalin with shRNA, then the nuclear transfer of the phosphorylated p53 is steady and operative, resulting in apoptotic death of cells. (C) In malignant cancer cells with high level of endogenous stress, p53 is phosphorylated and stable. Under such situations mortalin captures p53 in cytoplasm and inhibits its transcriptional activation, growth arrest and apoptotic functions. According to the proposed model, targetting the mortalin-p53 interaction could serve as a safe target for cancer therapeutics

phosphorylates I $\kappa \mathrm{B}$ proteins leading to their abasement and ensuing release of NF- $\kappa B$ (Fig. 2). This whole mechanism is classified under two broad pathways of NF- $\mathrm{KB}$ activation known as canonical pathway and non-canonical pathway. In the canonical pathway, which is prevalent in the cells stimulated with tumour necrosis factor $\alpha$ (TNF $\alpha$ ) or interleukin- $1 \beta$ (IL-1 $\beta$ ), initiates IKK $\beta$ which in turn phosphorylates IKB proteins at two $\mathrm{N}$-terminal serine residues. This phosphorylation targets I $\mathrm{KB}$ for polyubiquitination and successive proteasomal degradation, thus releasing $\mathrm{NF}-\kappa \mathrm{B}$. The non-canonical pathway of NF- $\kappa \mathrm{B}$ activation employs stimulation of receptors by BAFF, CD40 and lymphotoxin $\beta$ (LT $\beta$ ) ligand. Stimulation of these receptors activates the protein kinase NIK, which in turn activates IKK $\alpha$. IKK $\alpha$ on activation

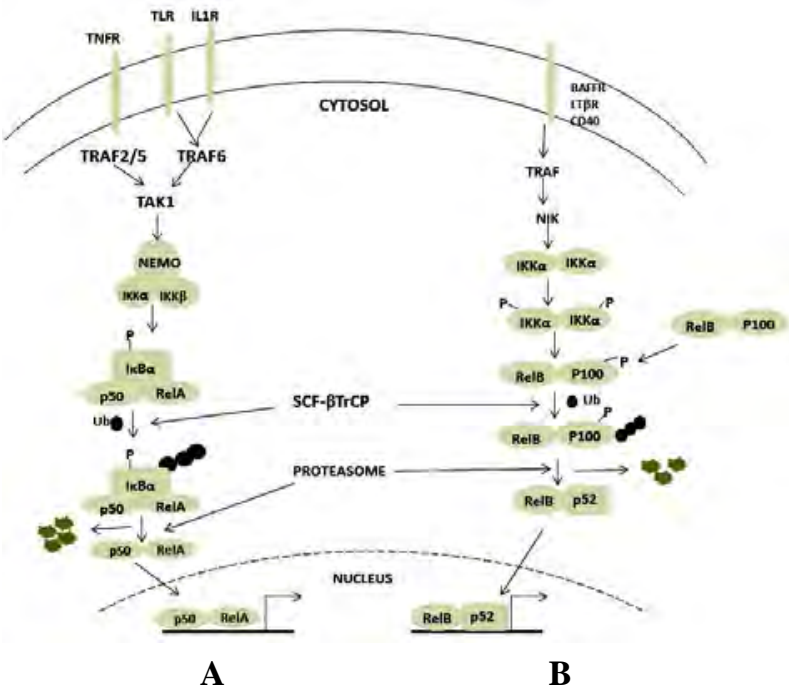

Fig. 2: The NF-KB signaling pathways: In the canonical pathway (A), different receptors like TNF receptors (TNFR), IL-1 receptors (IL-1R) or Toll-like receptors (TLR) on receiving their corresponding ligands activate the TRAF proteins and finally TAK1 kinase. TAK1 subsequently phosphorylates and activates IKK $\beta$. IKK $\beta$ after activation phosphorylates IKB, which finally leads to its polyubiquitination by SCF- $\beta$ TrCP ubiquitin ligase complex and ultimately gets degraded by the proteasome. NF- $\mathrm{KB}$ is a dimer, consisting of p50 (derived from p105) and RelA (commonly known as p65) sub-units. It enters the nucleus, binds to DNA through Rel-homology domain (RHD) and regulates the expression of target genes involved in inflammation, immunity and cell survival. In the non-canonical pathway $(B)$, receptors that belong to TNFR superfamily, like $B$ cell receptor for BAFF (BAFFR), activates NIK (kinase). NIK in turn phosphorylates IKK $\alpha$, which then leads to the phosphorylation of the NF-kB precursor, p100. After phosphorylation p100 is targeted to proteasomal degradation by polyubiquitination and ultimately forms the mature subunit p52. p52 along with Rel-B gets translocated to the nucleus to turn on target genes

phosphorylates p100 at two C-terminal serine residues leading to $\mathrm{I} \kappa \mathrm{B}$ degradation by proteasome, thus releasing NF- $\kappa \mathrm{B}$. (Various effects leading to constitutive activation of NF- $\kappa B$ in tumor cells have been mentioned in Table 2). Thus, hampering of NEMO/IKK $\beta$ complex assembly offers a mode of inhibition of NF- $\mathrm{KB}$ activation. Withaferin A is capable of inhibiting the genesis of active NEMO/IKK complex by either not allowing the formation of the complex or by disrupting the firmness of attachment of NEMO to IKK $\beta$ chains (Grover et al., 2010). 
However recently withaferin A was shown to potentiate apoptosis of tumour cells by suppression of NF- $\mathrm{KB}$ activation (Ichikawa et al., 2006).

\section{Inhibition of Proteasomal Activity}

Proteasomal activity of cells is required for tumour cell proliferation and to make cells resistant against drug. Therefore, inhibitors of proteasome mediated degradation pathway are required for cancer therapy and prevention. Ubiquitin is a 76-amino acid long protein covalently attached to the target protein during post- translational modifications. UPP (ubiquitin proteasome pathway) limits the activation of the transcription factor NF-kB, which is a regulatory protein playing central role in a variety of cellular processes. Since the predominant interaction of proteasomes occurs with endogenous proteins, the signal transduction pathway of transcription factor NF-kB can be obstructed by retarding proteasomes, which in turn inhibit the accomplishment of the cell cycle and mitotic multiplication of cancerous cells ultimately leading to cell death. Withaferin A acts as a proteasome inhibitor by suppressing its proteolytic activity which is due to N-terminal threonine (Thr1) residue hydroxyl group. This group is responsible for invoking the cleavage of peptides through nucleophilic attack, which is an irreversible covalent modification (Grover et al., 2010).

\section{Inhibition of HSP90/Cdc37 Complex}

Molecular chaperons are required for appropriate folding of many proteins, to remain biologically active. Heat Shock Protein (Hsp90) is one of the most abundant protein in eukaryotic systems. It manages proper folding and control in the cell environment. Hsp90 is essential for providing stability and activity to more than 200 client protein molecules including transmembrane tyrosine kinase proteins (Akt, Raf-1 and IKK), mutated signalling proteins (p53, Kit, FIt3and v-src), chimeric signalling proteins, steroid receptors and cell cycle regulators (cdk4, cdk6). Hsp90 maintains oncogenesis by preserving mutated or over expressed proteins and allowing their tumorigenic effect to become apparent. Hsp90 requires a number of co-chaperone assemblies for its activity. Cdc37 (cell division cycle protein 37 ) is one of the vital co-chaperones of Hsp90. Cdc37 acts as an adaptor and makes possible the maturation of kinase clients (Gray et al., 2008). Silencing of Cdc37 exhausts kinase clients and potentiates cell cycle arrest and apoptosis. Association of $\mathrm{Hsp} 90$ and its co-chaperone Cdc37 is interrupted by withaferin A by loosening the attachment of Hsp90 to Cdc37 (Grover et al., 2011). Also, withaferin A inhibits the function of Hsp90 and depletes other client proteins which take part in cancer disease state including Akt, CDK4, cyclin D and mutant p53. Furthermore, the inhibition of Hsp90 leads to deterioration of IKKs with diminished phosphorylation and degradation of IKB subsequently blocking NF- $\kappa \mathrm{B}$ translocation, NF- $\kappa \mathrm{B}$ DNA binding and gene transcription (Hertlein et al., 2010).

\section{Induction of Quinone Reductase}

Quinone reductase $(\mathrm{QR})$ is a cytosolic protein that assists the two-electron reduction of quinones and its derivatives, hence protects cells against oxidative stress. QR is induced by a wide range of antioxidants (Gordon et al., 1991) and some chemopreventive agents (Iqbal et al., 2003). The actual mechanism leading to the induction of cytosolic QR is unknown. However, the quinone reductase gene expression in turn is subjected to certain antioxidant inducers. The ARE element containing binding site for AP-1 (activator protein-1) and AP-2 (activator protein-2) is required for basal level expression of $\mathrm{QR}$ gene (Jaiswal, 2000; Begleiter and Fourie, 2004). It has been well established that the upregulation of QRhas been known to be a biomarker for cancer (Cuendet et al., 2006). QR functions majorly by diminishing the formation of ROS and it also works as a phase II detoxifying enzyme. Increased ROS level in cells leads to tumor initiation and promotion and may eventually lead to carcinogenesis. Additionally, QR regulates the stability of p53 by initiating some anticancer drugs, which eventually lead to apoptosis (Asher et al., 2001). Balance between carcinogen-activating phase I enzymes and phase II detoxifying enzymes is maintained by phytochemicals. It has been reported that 2,3-dihydro-3-methoxywithaphysacarpin, withaphysacarpin and 24,25-dihydrowithanolide D isolated from Physalis philadelphica, at the stage of DNA damage can prevent carcinogenesis 
Table 2: List of effects leading to constitutive activation of NF-KB in tumor cells

\begin{tabular}{lll}
\hline Effects & Tumor & References \\
\hline Inhibition of $C O X-2$ & Colorectal cancer & (Brown and DuBois, 2005) \\
Overexpression of NIK & Melanoma & (Dhawan et al., 2008) \\
Overexpression of Akt & Breast cancer & (Pianetti et al., 2001) \\
Overexpression of Raf & Multiple myeloma & (Keats et al., 2007) \\
Overexpression of LT- $\beta R$ & Melanoma & (Dhawan et al., 2008) \\
Overexpression of BAFFR & Kidney cancer & (Kohno et al., 2008) \\
TNF production & Breast cancer & (Braunstein et al., 2008) \\
IL-1 $\beta$ production & Acute myeloid leukemia & (Estrov et al., 2003) \\
I $\beta$ B $\alpha$ degradation & Gastric carcinoma & (Wu et al., 2008) \\
TRAF1 production & Cervical cancer & (Kato et al., 2008) \\
p53 mutations & Lung tumors & (Weisz et al., 2007)
\end{tabular}

(Kennelly et al., 1997). However, Singh and coworkers in 2011, reported that withaferin A isolated from Withania somnifera induces early generation of ROS leading to mitochondrial dysfunction in cancer cells (Singh et al., 2011).

\section{COX-2 Inhibitory Activity}

Cyclo-oxygenase-2 (COX-2) gene is thought to be involved in inflammation, mitogenesis and various signalling mechanisms. Several copies of Shawkamen's sequences are present in 3'- untranslated region (3'UTR) of $C O X-2$ which is responsible for enhanced Cox-2 mRNA degradation. Cox-2 transcripts are stabilized by proinflammatory cytokines. In contrast, corticosteroids destabilize Cox2 mRNA. Hence, in this way Cox-2 protein levels are regulated at transcriptional and translational level in normal cells. Whereas, it is reported that Cox-2 levels are high in case of tumorigenic cells, which is due to 5' region upstream of the $C O X-2$ gene. Cox2 protein is expressed on receiving a stimulus which could be oncogenes, growth factors, cytokines, chemotherapeutics and tumour promoters. Many trans factor binding motifs such as NF- $\mathrm{kb}$, IL-6, cAMP, CRE and HIF-I are present within promoter region of $C O X-2$ gene, which are involved in $C O X-2$ upregulation (Schmedtje et al., 1997). High levels of Cox-2 increase the risk of tumorigenesis. Cox-2 overexpression in cancerous cells could be through APC (member of Wnt signaling pathway). Wild type APC binds to $\beta$-catenin which is either membrane bound or free in cytosol. Cytosolic $\beta$-catenin is chopped by wild type APC under normal conditions, in conjunction of axin and glycogen synthase kinase-3 $\beta$ (GSK-3 $\beta$ ), whereas mutated APC cannot degrade cytosolic $\beta$-catenin. Free $\beta$-catenin gets translocated to nucleus and operates as a transcription factor in combination with T-cell factor-4 (TCF-4) complex. $\beta$-catenin in conjuction with TCF-4 binds to TCF-4 consensus site in target genes including $c$-myc, cyclin$D, P P A R \delta$ and $C O X-2$ along with the retinoid $\mathrm{X}$ receptor and a bound ligand, this in turn activates the target genes. $C O X-2$ inhibitors target and reduce both the possibility of ligand interaction (cyclo-oxygenasedependent) and the DNA binding of the complex (cyclo-oxygenase-independent) (Araki et al. 2003). TCF-4 binding sites are present within the $\mathrm{COX}-2$ promoter region. So, Cox-2 levels can be maintained by modulation between wild type APC activation or mutant APC induction. 


\section{Conclusion}

Withanolides are naturally occurring plant secondary metabolites with a $\delta$-lactone side chain made up of 28 carbons. These phytochemicals have great pharmacological value due to their special structural features. Out of the many potent properties, their anticancerous activity has drawn the interest of several researchers. Attempts have been made to enhance their production by in vitro culture techniques.

\section{References}

Araki Y, Okamura S, Hussain S P, Nagashima M, He P, Shiseki M, Miura K and Harris C C (2003) Regulation of cyclooxygenase-2 expression by the Wnt and ras pathways Cancer Res 63 728-734

Asher G, Lotem J, Cohen B, Sachs Land Shaul Y (2001) Regulation of p53 stability and p53-dependent apoptosis by NADH quinone oxidoreductase 1 Proc Natl Acad Sci U S A 98 1188-1193

Begleiter A and Fourie J (2004) Induction of NQO1 in cancer cells Methods Enzymol 382 320-351

Braunstein S, Formenti S C and Schneider R J (2008) Acquisition of stable inducible up-regulation of nuclear factor- $\kappa \mathrm{B}$ by tumor necrosis factor exposure confers increased radiation resistance without increased transformation in breast cancer cells Mol Cancer Res 6 78-88

Brown J R and DuBois R N (2005) COX-2: a molecular target for colorectal cancer prevention J Clin Oncol 232840 2855

Chaurasiya N, Sangwan N, Sabir F, Misra L and Sangwan R (2012) Withanolide biosynthesis recruits both mevalonate and DOXP pathways of isoprenogenesis in Ashwagandha Withania somnifera L. (Dunal) Plant Cell Reports 31 18891897

Chaurasiya N D, Sangwan R S, Misra L N, Tuli R and Sangwan N S (2009) Metabolic clustering of a core collection of Indian ginseng Withania somnifera Dunal through DNA, isoenzyme, polypeptide and withanolide profile diversity Fitoterapia 80 496-505

Chen L X, He H and Qiu F (2011) Natural withanolides: an overview Nat Prod Rep 28 705-740

Cuendet M, Oteham C P, Moon R C and Pezzuto J M (2006) Quinone Reductase Induction as a Biomarker for Cancer Chemoprevention J natural products 69 460-463

Dhawan P, Su Y, Thu Y M, Yu Y, Baugher P, Ellis D L, Sobolik-
Withanolides have achieved an important place in the world of drugs. Withanolides either independently or in conjunction with other signalling molecules regulate the expression of many carcinogenic genes. Hence, it prevents tumorigenesis by regulating the cell cycle. Action mechanism of these compounds for their antiproliferative effect involves several processes including induction of apoptosis by p53 genes. Overall, withanolides can act as a safe source of antitumour medicine.

Delmaire T, Kelley M, Cheung T C, Ware C F and Richmond A (2008) The lymphotoxin- $\beta$ receptor is an upstream activator of NF- $\mathrm{KB}-$ mediated transcription in melanoma cells J Biol Chem 283 15399-15408

Estrov Z, Shishodia S, Faderl S, Harris D, Van Q, Kantarjian H M, Talpaz M and Aggarwal B B (2003) Resveratrol blocks interleukin-1 $\beta$-induced activation of the nuclear transcription factor $\mathrm{NF}-\kappa \mathrm{B}$, inhibits proliferation, causes S-phase arrest, and induces apoptosis of acute myeloid leukemia cells Blood 102 987-995

Gordon G B, Prochaska H J and Yang L Y (1991) Induction of $\mathrm{NAD}(\mathrm{P}) \mathrm{H}$ :quinone reductase in human peripheral blood lymphocytes Carcinogenesis 12 2393-2396

Gray P J, Jr., Prince T, Cheng J, Stevenson M A and Calderwood S K (2008) Targeting the oncogene and kinome chaperone CDC37 Nat Rev Cancer 8 491-495

Grover A, Priyandoko D, Gao R, Shandilya A, Widodo N, Bisaria V S, Kaul S C, Wadhwa R and Sundar D (2012) Withanone binds to mortalin and abrogates mortalin-p53 complex: computational and experimental evidence Int J Biochem Cell Biol 44 496-504

Grover A, Samuel G, Bisaria V S and Sundar D (2013) Enhanced withanolide production by overexpression of squalene synthase in Withania somnifera $J$ Bioscience and Bioengineering 115 680-685

Grover A, Shandilya A, Agrawal V, Pratik P, Bhasme D, Bisaria V S and Sundar D (2011) Hsp90/Cdc37 chaperone/cochaperone complex, a novel junction anticancer target elucidated by the mode of action of herbal drug Withaferin A BMC Bioinformatics 15 1471-2105

Grover A, Shandilya A, Bisaria V S and Sundar D (2010) Probing the anticancer mechanism of prospective herbal drug Withaferin A on mammals: a case study on human and bovine proteasomes BMC Genomics 2 1471-2164

Grover A, Shandilya A, Punetha A, Bisaria V S and Sundar D (2010) Inhibition of the NEMO/IKK $\beta$ association complex 
formation, a novel mechanism associated with the NF- $\kappa \mathrm{B}$ activation suppression by Withania somnifera's key metabolite withaferin A BMC Genomics 2 1471-2164

Hertlein E, Wagner A J, Jones J, Lin T S, Maddocks K J, Towns W H, 3rd, Goettl V M, Zhang X, Jarjoura D, Raymond C A, West D A, Croce C M, Byrd J C and Johnson A J (2010) 17-DMAG targets the nuclear factor- $\mathrm{kB}$ family of proteins to induce apoptosis in chronic lymphocytic leukemia: clinical implications of HSP90 inhibition Blood $11645-53$

Ichikawa H, Takada Y, Shishodia S, Jayaprakasam B, Nair M G and Aggarwal B B (2006) Withanolides potentiate apoptosis, inhibit invasion, and abolish osteoclastogenesis through suppression of nuclear factor- $\kappa \mathrm{B}(\mathrm{NF}-\kappa \mathrm{B})$ activation and NF- $\mathrm{\kappa B}$-regulated gene expression $\mathrm{Mol}$ Cancer Ther 5 1434-1445

Iqbal M, Sharma S D, Okazaki Y, Fujisawa M and Okada S (2003) Dietary supplementation of curcumin enhances antioxidant and phase II metabolizing enzymes in ddY male mice: possible role in protection against chemical carcinogenesis and toxicity Pharmacol Toxicol 92 33-38

Jaiswal A K (2000) Regulation of genes encoding NAD(P)H:quinone oxidoreductases Free Radic Biol Med 29 254-262

Kaiser H, Richter U, Keiner R, Brabant A, Hause B and Drager B (2006) Immunolocalisation of two tropinone reductases in potato (Solanum tuberosum L.) root, stolon, and tuber sprouts Planta 225 127-137

Kato T, Jr., Gotoh Y, Hoffmann A and Ono Y (2008) Negative regulation of constitutive NF- $\mathrm{\kappa B}$ and JNK signaling by PKN1-mediated phosphorylation of TRAF1 Genes Cells 13 509-520

Keats J J, Fonseca R, Chesi M, Schop R, Baker A, Chng W J, Van Wier S, Tiedemann R, Shi C X, Sebag M, Braggio E, Henry T, Zhu Y X, Fogle H, Price-Troska T, Ahmann G, Mancini C, Brents L A, Kumar S, Greipp P, Dispenzieri A, Bryant B, Mulligan G, Bruhn L, Barrett M, Valdez R, Trent J, Stewart A K, Carpten J and Bergsagel P L (2007) Promiscuous mutations activate the noncanonical NF- $\mathrm{\kappa B}$ pathway in multiple myeloma Cancer Cell 12 131-144

Kennelly E J, Gerhäuser C, Song L L, Graham J G, Beecher C W W, Pezzuto J M and Kinghorn A D (1997) Induction of Quinone Reductase by Withanolides Isolated from Physalis philadelphica (Tomatillos) J Agricultural and Food Chemistry 45 3771-3777

Kim O, Bang K, Kim Y, Hyun D, Kim M and Cha S (2009) Upregulation of ginsenoside and gene expression related to triterpene biosynthesis in ginseng hairy root cultures elicited by methyl jasmonate Plant Cell, Tissue and Organ Culture (PCTOC) 98 25-33

Kinghorn A D, Su B N, Jang D S, Chang L C, Lee D, Gu J Q, Carcache-Blanco E J, Pawlus A D, Lee S K, Park E J, Cuendet M, Gills J J, Bhat K, Park H S, Mata-Greenwood E, Song L L, Jang M and Pezzuto J M (2004) Natural inhibitors of carcinogenesis Planta Med 70 691-705

Kohno T, Daa T, Otani H, Shimokawa I, Yokoyama S and Matsuyama T (2008) Aberrant expression of BAFF receptor, a member of the tumor necrosis factor receptor family, in malignant cells of nonhematopoietic origins Genes Cells 13 1061-1073

Lee J H, Yoon Y H, Kim H Y, Shin D H, Kim D U, Lee I J and Kim K U (2002) Cloning and expression of squalene synthase cDNA from hot pepper (Capsicum annuum L.) Mol Cells 13 436-443

Lu W J, Lee N P, Kaul S C, Lan F, Poon R T, Wadhwa R and Luk J M (2011) Mortalin-p53 interaction in cancer cells is stress dependent and constitutes a selective target for cancer therapy Cell Death Differ 18 1046-1056

Mirjalili H M, Fakhr-Tabatabaei S M, Bonfill M, Alizadeh H, Cusido R M, Ghassempour A and Palazon J (2009) Morphology and withanolide production of Withania coagulans hairy root cultures Engineering in Life Sciences 9 197-204

Mishra L, Singh B and Dagenias S (2000) Scientific basis for the therapeutic use of Withania somnifera (ashwagandha): a review Alternative Medicine Review 5 334-336

Murthy H N, Dijkstra C, Anthony P, White D A, Davey M R, Power J B, Hahn E J and Paek K Y (2008) Establishment of Withania somnifera hairy root cultures for the production of withanolide A J Integr Plant Biol 50 975981

Pianetti S, Arsura M, Romieu-Mourez R, Coffey R J and Sonenshein G E (2001) Her-2/neu overexpression induces $\mathrm{NF}-\kappa \mathrm{B}$ via a PI3-kinase/Akt pathway involving calpainmediated degradation of I $\mathrm{KB}-\alpha$ that can be inhibited by the tumor suppressor PTEN Oncogene 20 1287-1299

Radman R, Saez T, Bucke C and Keshavarz T (2003) Elicitation of plants and microbial cell systems Biotechnol Appl Biochem 37 91-102

Raffo A J, Kim A L and Fine R L (2000) Formation of nuclear $\mathrm{Bax} / \mathrm{p} 53$ complexes is associated with chemotherapy induced apoptosis Oncogene 19 6216-6228

Sangwan R S, Chaurasiya N D, Lal P, Misra L, Tuli R and Sangwan N S (2008) Withanolide A is inherently de novo biosynthesized in roots of the medicinal plant Ashwagandha (Withania somnifera) Physiologia Plantarum 133 278-287 
Santos P A G, Figueiredo A C, Oliveira M M, Barroso J G, Pedro L G, Deans S G and Scheffer J J C (2005) Growth and essential oil composition of hairy root cultures of Levisticum officinale WDJ Koch (lovage) Plant Science 168 1089-1096

Schmedtje J F, Jr., Ji Y S, Liu W L, DuBois R N and Runge M S (1997) Hypoxia induces cyclooxygenase-2 via the NF- $\kappa B$ p65 transcription factor in human vascular endothelial cells J Biol Chem 272 601-608

Singh N, Verma P, Pandy B R and Gilca M G (2011) Role of Withania somnifera in prevention and treatment of cancer: an overview International Journal of Pharmaceutical Sciences and Drug Research 3 274-279

Sivanandhan G, Kapil Dev G, Jeyaraj M, Rajesh M, Arjunan A, Muthuselvam M, Manickavasagam M, Selvaraj N and Ganapathi A (2013) Increased production of withanolide $\mathrm{A}$, withanone, and withaferin $\mathrm{A}$ in hairy root cultures of Withania somnifera (L.) Dunal elicited with methyl jasmonate and salicylic acid Plant Cell, Tissue and Organ Culture (PCTOC) 114 121-129
Tozawa R-i, Ishibashi S, Osuga J-i, Yagyu H, Oka T, Chen Z, Ohashi K, Perrey S, Shionoiri F, Yahagi N, Harada K, Gotoda T, Yazaki Y and Yamada N (1999) Embryonic Lethality and Defective Neural Tube Closure in Mice Lacking Squalene Synthase J Biological Chemistry 274 30843-30848

Weisz L, Damalas A, Liontos M, Karakaidos P, Fontemaggi G, Maor-Aloni R, Kalis M, Levrero M, Strano S, Gorgoulis V G, Rotter V, Blandino G and Oren M (2007) Mutant p53 enhances nuclear factor $\kappa \mathrm{B}$ activation by tumor necrosis factor $\alpha$ in cancer cells Cancer Res 67 2396-2401

Wu H, Li W, Wang T, Shu Y and Liu P (2008) Paeoniflorin suppress NF- $\kappa$ B activation through modulation of IкB $\alpha$ and enhances 5-fluorouracil-induced apoptosis in human gastric carcinoma cells Biomed Pharmacother 62 659-666

Zhao D, Fu C, Chen Y and Ma F (2004) Transformation of Saussurea medusa for hairy roots and jaceosidin production Plant Cell Reports 23 468-474

Zhao J, Davis L C and Verpoorte R (2005) Elicitor signal transduction leading to production of plant secondary metabolites Biotechnol Adv 23 283-333. 